\title{
Second order natural Lagrangians on coframe bundles
}

\author{
Ján Brajerčik and Milan Demko
}




\title{
SECOND ORDER NATURAL LAGRANGIANS ON COFRAME BUNDLES
}

\author{
JÁN BRAJERČĆÍK AND MILAN DEMKO
}

\begin{abstract}
We study the structure of second order natural Lagrangians on the bundle of linear coframes $F^{*} X$ over an $n$-dimensional manifold $X$. They are identified with the corresponding differential invariants which can be obtained by the factorization method. We give an explicit description of these differential invariants in terms of their bases. For construction of natural Lagrangians, the canonical odd $n$-form on $F^{*} X$ is also introduced.
\end{abstract}

2000 Mathematics Subject Classification: 53A55, 58A10, 58A20

Keywords: natural Lagrangian, coframe, differential invariant, equivariant mapping, differential group

\section{INTRODUCTION AND PRELIMINARIES}

The aim of this paper is to characterize all global, second order Lagrangians on coframe bundles $F^{*} X$, invariant with respect to diffeomorphisms of $X$. Such Lagrangians are called natural. Natural Lagrangians on coframe bundles play an important role in several theories, such as teleparallel description of gravity (see, e. g., [11]). A characteristic property of a natural Lagrangian is that it is a differential invariant. The domain of definition of second order natural Lagrangians on coframe bundles, the bundle $J^{2} F^{*} X$, can equivalently be understood as the type fibre of $J^{2} F^{*} X$.

As usual, we denote by $\mathbb{R}$ the field of real numbers. The $r$-th differential group $L_{n}^{r}$ of $\mathbb{R}^{n}$ is the Lie group of invertible $r$-jets with source and target at the origin $0 \in \mathbb{R}^{n}$. The group multiplication in $L_{n}^{r}$ is defined by the composition of jets. The first (second) canonical coordinates are denoted by $a_{j_{1}}^{i}, a_{j_{1} j_{2}}^{i}, \ldots, a_{j_{1} j_{2} \ldots j_{r}}^{i}\left(b_{j_{1}}^{i}\right.$, $b_{j_{1} j_{2}}^{i}, \ldots, b_{j_{1} j_{2} \ldots j_{r}}^{i}$ ), where $1 \leq i \leq n, 1 \leq j_{1} \leq j_{2} \leq \ldots \leq j_{r} \leq n$. The canonical jet projection of $L_{n}^{r}$ onto $L_{n}^{1}$ is a Lie group homomorphism. Denoting by $K_{n}^{r}$ its kernel, we can represent the differential group $L_{n}^{r}$ as a semi-direct product of $L_{n}^{1}$ and $K_{n}^{r}, L_{n}^{r}=L_{n}^{1} \times_{s} K_{n}^{r}$. For generalities on spaces of jets and their mappings, differential groups, their actions, etc., we refer to $[6,10]$.

By a left $G$-manifold we mean a smooth manifold endowed with a left action of a Lie group $G$. Let $P$ and $Q$ be two left $L_{n}^{r}$-manifolds. A smooth mapping $F: P \rightarrow$

The first author was supported by the Czech Grant Agency, Grant No. 201/09/0981. 
$Q$ is called a differential invariant if it is $L_{n}^{r}$-equivariant, i.e., $F(g \cdot p)=g \cdot F(p)$ for all $g \in L_{n}^{r}$ and $p \in P$.

If $L_{n}^{r}$ acts on manifold $Q$ from the left via its subgroup $L_{n}^{1}$, and $F: P \rightarrow Q$ is a differential invariant, then the restriction of the group action of $L_{n}^{r}$ to $K_{n}^{r}$ gives the condition $F(g \cdot p)=F(p)$. This is the basic idea of the orbit reduction method, first time used by Krupka in [7], which utilizes the semi-direct product of $L_{n}^{r}$ to compute the differential invariants.

Let us consider a left $L_{n}^{1}$-manifold $Q$, and denote by $T_{n}^{r} Q$ the manifold of $r$-jets with source $0 \in \mathbb{R}^{n}$ and target in $Q$. According to the general theory of prolongations of left $G$-manifolds, $T_{n}^{r} Q$ has a (canonical) structure of a left $L_{n}^{r+1}$-manifold. To define this structure, denote by $t_{x}$ the translation of $\mathbb{R}^{n}$ defined by $t_{x}(y)=y-x$. Consider elements $q \in T_{n}^{r} Q, q=J_{0}^{r} \gamma$, and $a \in L_{n}^{r+1}, a=J^{r+1} \alpha$. If we denote $\bar{\alpha}_{x}=t_{x} \circ \alpha \circ t_{-\alpha^{-1}(x)}$, setting $\bar{\alpha}(x)=J_{0}^{1} \bar{\alpha}_{x}$ we get an element of the group $L_{n}^{1}$. Then the formula

$$
a \cdot q=J_{0}^{r}\left(\bar{\alpha} \cdot\left(\gamma \circ \alpha^{-1}\right)\right)
$$

defines a left action of the differential group $L_{n}^{r+1}$ on $T_{n}^{r} Q$. The formula (1.1) is usually called the prolongation formula for the action of the group $L_{n}^{1}$ on $Q$. The left $L_{n}^{r+1}$-manifold $T_{n}^{r} Q$ is called the $r$-jet prolongation of the left $L_{n}^{1}$-manifold $Q$.

Let $X$ be an $n$-dimensional manifold. By an $r$-frame at a point $x \in X$ we mean an invertible $r$-jet with source $0 \in \mathbb{R}^{n}$ and target $x$. The set of $r$-frames together with its natural structure of a principal $L_{n}^{r}$-bundle with base $X$ is denoted by $F^{r} X$, and is called the bundle of $r$-frames over $X$. For $r=1$, we get the bundle of linear frames, and write $F^{1} X=F X$. If $Q$ is a left $L_{n}^{1}$-manifold, then the bundle with type fibre $Q$, associated with $F X$ is denoted by $F_{Q} X$. Formula (1.1) defines on $J^{r} F_{Q} X$ the structure of a fibre bundle with type fibre $T_{n}^{r} Q$, associated with the bundle $F^{r+1} X$ (see, e. g., [9]).

An $r$-coframe at $x \in X$ is an invertible $r$-jet with source $x$ and target $0 \in \mathbb{R}^{n}$. If $r=1$, we speak of linear coframes. We denote by $F^{*} X$ the set of all linear coframes at all $x \in X$. The right action of $L_{n}^{1}$ on $F^{*} X$ is given by

$$
F^{*} X \times L_{n}^{1} \ni\left(J_{x}^{1} \xi, J_{0}^{1} \alpha\right) \mapsto J_{x}^{1} \xi \cdot J_{0}^{1} \alpha=J_{x}^{1}\left(\alpha^{-1} \circ \xi\right) \in F^{*} X .
$$

With this action, $F^{*} X$ is a right principal $L_{n}^{1}$-bundle.

$F^{*} X$ can also be considered as a fibre bundle with type fibre $L_{n}^{1}$ associated with $L_{n}^{1}$-bundle $F X$, i.e., we have an identification $F^{*} X=F_{L_{n}^{1}} X$. Induced left action of the group $L_{n}^{1}$ on the type fibre $L_{n}^{1}$, is given by

$$
L_{n}^{1} \times L_{n}^{1} \ni\left(J_{0}^{1} \alpha, J_{0}^{1} \eta\right) \mapsto J_{0}^{1}\left(\eta \circ \alpha^{-1}\right) \in L_{n}^{1} .
$$

In the canonical coordinates $\left(p_{j}^{i}\right)$ on the type fibre $L_{n}^{1}$ of $F^{*} X,(1.3)$ is expressed as

$$
p_{j}^{i}\left(J_{0}^{1}\left(\eta \circ \alpha^{-1}\right)\right)=p_{s}^{i}\left(J_{0}^{1} \eta\right) a_{j}^{s}\left(J_{0}^{1} \alpha^{-1}\right)=p_{s}^{i}\left(J_{0}^{1} \eta\right) b_{j}^{s}\left(J_{0}^{1} \alpha\right),
$$


or simply by

$$
\bar{p}_{j}^{i}=p_{s}^{i} b_{j}^{s}
$$

(1.5) is called the coframe action of $L_{n}^{1}$ on itself.

$J^{r} F^{*} X$ denotes the $r$-jet prolongation of $F^{*} X$. Since $F^{*} X=F_{L_{n}^{1}} X, J^{r} F^{*} X$ has the structure of a fibre bundle with type fibre $T_{n}^{r} L_{n}^{1}$, associated with $F^{r+1} X$. The action of $L_{n}^{r+1}$ on $T_{n}^{r} L_{n}^{1}$ can be obtained by prolongation of the action (1.4) of $L_{n}^{1}$ on $L_{n}^{1}$ using the formula (1.1).

Let us consider a left action of the general linear group $L_{n}^{1}$ on the real line $\mathbb{R}$ defined by $L_{n}^{1} \times \mathbb{R} \ni(a, t) \mapsto\left|\operatorname{det} a^{-1}\right| \cdot t \in \mathbb{R}$. The real line, endowed with this action, is an $L_{n}^{1}$-manifold, denoted by $\widetilde{\mathbb{R}}$. A differential invariant with values in $\widetilde{\mathbb{R}}$ is called a scalar invariant. General result on the structure of natural Lagrangians says that, given $L_{n}^{1}$-manifold $Q$, there is a one-to-one correspondence between natural Lagrangians on $J^{r} F_{Q} X$ and differential invariants $d: T_{n}^{r} Q \rightarrow \widetilde{\mathbb{R}}$ (see, e. g., [9]). This means that for finding second order natural Lagrangians of coframe bundles it is sufficient to describe all scalar invariants of $T_{n}^{2} L_{n}^{1}$ associated with the coframe action (1.5).

\section{BASIS OF THE SECOND ORDER INVARIANTS}

We are interested in differential invariants $F: P \rightarrow Q$ with values in $L_{n}^{1}$-manifold $Q$, which can be viewed as manifold with action of $L_{n}^{r}$ via its subgroup $L_{n}^{1}$. In such case, each differential invariant $F: P \rightarrow Q$ has the form $F=f \circ \pi$, where $\pi$ : $P \rightarrow P / K_{n}^{r}$ is the canonical projection onto the orbit space, and $f: P / K_{n}^{r} \rightarrow Q$ is a uniquely determined $L_{n}^{1}$-equivariant mapping (see [10]).

Indeed, in this scheme $P / K_{n}^{r}$ is considered with the quotient topology, but it is not necessarily a smooth manifold. The quotient projection $\pi$ is continuous but not necessarily smooth. If $P / K_{n}^{r}$ has a smooth structure such that $\pi$ is a submersion, we call $\pi$ the basis of differential invariants on $P$. The general concepts on equivariant mappings, related with a normal subgroup of a Lie group, and corresponding assertions with the proofs can be found in $[4,7]$.

Our aim is to find a basis of differential invariants on $T_{n}^{2} L_{n}^{1}$. We use the expression of the action of $L_{n}^{3}$ on $T_{n}^{2} L_{n}^{1}$ (see [3]), obtained as prolongation of the coframe action of $L_{n}^{1}$ on $L_{n}^{1}(1.5)$,

$$
\begin{aligned}
& \bar{p}_{j}^{i}=p_{s}^{i} b_{j}^{s}, \\
& \bar{p}_{j, k}^{i}=p_{s, t}^{i} b_{k}^{t} b_{j}^{s}+p_{s}^{i} b_{j k}^{s}, \\
& \bar{p}_{j, k l}^{i}=p_{s, t u}^{i} b_{l}^{u} b_{k}^{t} b_{j}^{s}+p_{s, t}^{i}\left(b_{k l}^{t} b_{j}^{s}+b_{k}^{t} b_{j l}^{s}+b_{l}^{t} b_{j k}^{s}\right)+p_{s}^{i} b_{j k l}^{s} .
\end{aligned}
$$


Restricting to the kernel $K_{n}^{3}$ of the projection $\pi_{n}^{3,1}: L_{n}^{3} \rightarrow L_{n}^{1}$ we obtain the group action of $K_{n}^{3}$ on $T_{n}^{2} L_{n}^{1}$ induced by the coframe action (see [3])

$$
\begin{aligned}
& \bar{p}_{j}^{i}=p_{j}^{i}, \\
& \bar{p}_{j, k}^{i}=p_{j, k}^{i}+p_{s}^{i} b_{j k}^{s}, \\
& \bar{p}_{j, k l}^{i}=p_{j, k l}^{i}+p_{j, t}^{i} b_{k l}^{t}+p_{s, k}^{i} b_{j l}^{s}+p_{s, l}^{i} b_{j k}^{s}+p_{s}^{i} b_{j k l}^{s} .
\end{aligned}
$$

We denote by $q_{j}^{i}$ the inverse matrix of the matrix $p_{j}^{i}$; thus, $q_{j}^{i}: T_{n}^{2} L_{n}^{1} \rightarrow \mathbb{R}$ are functions such that $q_{s}^{i} p_{j}^{s}=\delta_{j}^{i}$, where $\delta_{j}^{i}$ denotes the Kronecker symbol. Symmetrization (antisymmetrization) in some indices $j, k, l, \ldots$ is denoted by writing a bar (a tilde) over these indices, i.e., by writing $\bar{j}, \bar{k}, \bar{l}, \ldots(\tilde{j}, \tilde{k}, \tilde{l}, \ldots)$.

We also introduce the following functions on $T_{n}^{2} L_{n}^{1}$ :

$$
\begin{aligned}
& I_{j, k}^{i}\left(p_{b}^{a}, p_{b, c}^{a}, p_{b, c d}^{a}\right)=q_{m}^{i} p_{\tilde{j}, \tilde{k}}^{m}, \\
& I_{j, k l}^{i}\left(p_{b}^{a}, p_{b, c}^{a}, p_{b, c d}^{a}\right)=q_{m}^{i} p_{\tilde{j}, \tilde{k} l}^{m}+q_{m}^{i} q_{t}^{s}\left(p_{\tilde{s}, \tilde{j}}^{m} p_{\bar{k}, \bar{l}}^{t}-p_{\tilde{s}, \tilde{k}}^{m} p_{\bar{j}, \bar{l}}^{t}\right) .
\end{aligned}
$$

Lemma 1. $K_{n}^{3}$-orbits in $T_{n}^{2} L_{n}^{1}$ induced by the coframe action of $L_{n}^{1}$ on $L_{n}^{1}$ are defined by the equations

$$
p_{j}^{i}=c_{j}^{i}, \quad I_{j, k}^{i}\left(p_{b}^{a}, p_{b, c}^{a}, p_{b, c d}^{a}\right)=c_{j, k}^{i}, \quad I_{j, k l}^{i}\left(p_{b}^{a}, p_{b, c}^{a}, p_{b, c d}^{a}\right)=c_{j, k l}^{i},
$$

where $c_{j}^{i}, c_{j, k}^{i}, c_{j, k l}^{i} \in \mathbb{R}$ are constants satisfying $\operatorname{det} c_{j}^{i} \neq 0, c_{j, k}^{i}+c_{k, j}^{i}=0$, $c_{j, k l}^{i}+c_{k, j l}^{i}=0$, and $c_{j, k l}^{i}-c_{j, l k}^{i}=0$.

Proof. Since the coordinates $b_{j k}^{s}$ are symmetric in $j, k$, from the action (2.2) we can write $b_{j k}^{s}=q_{i}^{s}\left(\bar{p}_{\bar{j}, \bar{k}}^{i}-p_{\bar{j}, \bar{k}}^{i}\right)$, and substituting this expression of $b_{j k}^{s}$ in (2.2), we get

$$
q_{i}^{s}\left(\bar{p}_{j, k}^{i}-p_{j, k}^{i}\right)=q_{i}^{s}\left(\bar{p}_{\bar{j}, \bar{k}}^{i}-p_{\bar{j}, \bar{k}}^{i}\right) .
$$

Now we compare the tensor on the left hand side with its symmetric part. Using $\bar{q}_{i}^{s}=$ $q_{i}^{s}$, it gives us $\bar{q}_{i}^{s} \bar{p}_{\tilde{j}, \tilde{k}}^{i}=q_{i}^{s} p_{\tilde{j}, \tilde{k}}^{i}$. Thus, for the functions $I_{j, k}^{s}$, defined by (2.3), we have $I_{j, k}^{s}\left(\bar{p}_{b}^{a}, \bar{p}_{b, c}^{a}, \bar{p}_{b, c d}^{a}\right)=I_{j, k}^{s}\left(p_{b}^{a}, p_{b, c}^{a}, p_{b, c d}^{a}\right)$. If we denote $\Delta_{j k l}^{s}=q_{i}^{s}\left(\bar{p}_{j, k l}^{i}-\right.$ $p_{j, k l}^{i}-p_{j, t}^{i} b_{k l}^{t}-p_{t, k}^{i} b_{j l}^{t}-p_{t, l}^{i} b_{j k}^{t}$ ), expressing $b_{j k l}^{s}$ from (2.2), we analogously obtain that $\Delta_{j k l}^{s}=\Delta_{\bar{j} \bar{k} \bar{l}}^{s}$. Using the Young decomposition of the tensor $\Delta_{j k l}^{s}$, this equation is equivalent to $I_{j, k l}^{s}\left(\bar{p}_{b}^{a}, \bar{p}_{b, c}^{a}, \bar{p}_{b, c d}^{a}\right)=I_{j, k l}^{s}\left(p_{b}^{a}, p_{b, c}^{a}, p_{b, c d}^{a}\right)$. Therefore the functions $I_{j, k}^{s}$ and $I_{j, k l}^{s}$ are invariant with respect to the action (2.2). Finally, it is easy to see that the functions $I_{j, k}^{i}, I_{j, k l}^{i}$ are antisymmetric in the indices $j, k$, and the functions $I_{j, k l}^{i}$ are symmetric in the indices $k, l$. 
Corollary 1. The mappings $p_{j}^{i}, I_{j, k}^{i}, I_{j, k l}^{i}$ represent a basis of second order invariants of coframes with values in left $L_{n}^{1}$-manifold.

\section{SCALAR INVARIANTS OF COFRAMES}

In order to obtain scalar invariants of $L_{n}^{3}$ on $T_{n}^{2} L_{n}^{1}$ it is sufficient to consider $L_{n}^{1}$ equivariant mappings defined on $T_{n}^{2} L_{n}^{1} / K_{n}^{3}$ (see [1]). Let us define the functions $l_{j, k}^{i}, d_{j, k l}^{i}$, on $T_{n}^{2} L_{n}^{1}$, by

$$
\begin{aligned}
& \mathcal{l}_{j, k}^{i}=q_{j}^{s} q_{k}^{t} p_{\tilde{s}, \tilde{t}}^{i}, \\
& \mathcal{l}_{j, k l}^{i}=q_{j}^{s} q_{k}^{t} q_{l}^{u}\left(p_{\tilde{s}, \tilde{t} u}^{i}+q_{m}^{v}\left(p_{\tilde{v}, \tilde{s}}^{i} p_{\bar{t}, \bar{u}}^{m}-p_{\tilde{v}, \tilde{t}}^{i} p_{\bar{s}, \bar{u}}^{m}\right)\right)
\end{aligned}
$$

Theorem. (a) The functions $d_{j, k}^{i}, d_{j, k l}^{i}$ on $T_{n}^{2} L_{n}^{1}$ are invariant with respect to the prolonged coframe action.

(b) Any function on $T_{n}^{2} L_{n}^{1}$, invariant with respect to the prolonged coframe action, is a differentiable function of the functions $d_{j, k}^{i}$, and $d_{j, k l}^{i}$.

Proof. (a) The group $L_{n}^{1} \simeq L_{n}^{3} / K_{n}^{3}$ acts in orbit space $T_{n}^{2} L_{n}^{1} / K_{n}^{3}$, with coordinates $I_{j, k}^{i}$ and $I_{j, k l}^{i}$, by

$$
\bar{I}_{j, k}^{i}=a_{r}^{i} b_{j}^{s} b_{k}^{t} I_{s, t}^{r}, \quad \bar{I}_{j, k l}^{i}=a_{r}^{i} b_{j}^{s} b_{k}^{t} b_{l}^{u} I_{s, t u}^{r} .
$$

Using relations $a_{r}^{i}=\bar{q}_{l}^{i} p_{r}^{l}$, and $b_{j}^{s}=q_{m}^{s} \bar{p}_{j}^{m}$, obtained from (1.5), in (3.2), we have

$$
\bar{p}_{i}^{a} \bar{q}_{b}^{j} \bar{q}_{c}^{k} \bar{I}_{j, k}^{i}=p_{r}^{a} q_{b}^{s} q_{c}^{t} I_{s, t}^{r}, \quad \bar{p}_{i}^{a} \bar{q}_{b}^{j} \bar{q}_{c}^{k} \bar{q}_{d}^{l} \bar{I}_{j, k l}^{i}=p_{r}^{a} q_{b}^{s} q_{c}^{t} q_{d}^{u} I_{s, t u}^{r},
$$

which describes $L_{n}^{1}$-invariant objects in $T_{n}^{2} L_{n}^{1} / K_{n}^{3}$. Applying (2.3), we get

$$
p_{r}^{i} q_{j}^{s} q_{k}^{t} I_{s, t}^{r}=d_{j, k}^{i}, \quad p_{r}^{i} q_{j}^{s} q_{k}^{t} q_{l}^{u} I_{s, t u}^{r}=d_{j, k l}^{i},
$$

where $d_{j, k}^{i}, d_{j, k l}^{i}$ are given by (3.1).

(b) The statement follows from the invariance theory.

Lemma 2. The function $d_{0}: T_{n}^{2} L_{n}^{1} \ni q \mapsto d_{0}(q)=\left|\operatorname{det} q_{j}^{i}(q)\right| \in \widetilde{\mathbb{R}}$ is $a$ differential invariant.

Proof. Obviously, the function $d_{0}$ is smooth, and for every $a \in L_{n}^{3}$, and every $q \in T_{n}^{2} L_{n}^{1}$ we have $d_{0}(a \cdot q)=\left|\operatorname{det} a^{-1}\right| \cdot d_{0}(q)$.

Corollary 2. Every differential invariant $d: T_{n}^{2} L_{n}^{1} \rightarrow \widetilde{\mathbb{R}}$ is the product of some differentiable function of $d_{j, k}^{i}, d_{j, k l}^{i}$, and the function $d_{0}$. 


\section{SECOND ORDER NATURAL LAGRANGIANS OF COFRAMES}

Our aim in this Section is to characterize all Lagrangians on $J^{2} F^{*} X$, invariant with respect to all diffeomorphisms of $X$. First, we recall the main concepts to this purpose.

We present basic definitions in full generality (for odd base forms). If the underlying manifold $X$ is orientable, odd base forms may be replaced by ordinary forms. The concept of volume form is needed for integration on not necessarily orientable manifold.

Any chart $(U, \varphi), \varphi=\left(x^{i}\right)$, on $X$, induces the fibred chart $(V, \psi), \psi=\left(x^{i}, z_{j}^{i}\right)$, on $F^{*} X$. By setting $z_{j}^{i} w_{k}^{j}=\delta_{k}^{i}$ we define another coordinates $w_{k}^{j}$ on $F^{*} X$. With a chart $(V, \psi)$ we also associate the object

$$
\tilde{\omega}_{(V, \psi)}=\left|\operatorname{det} z_{j}^{i}\right| \cdot \tilde{\varphi} \otimes d x^{1} \wedge d x^{2} \wedge \ldots \wedge d x^{n},
$$

where $\tilde{\varphi}$ is a field of odd scalars on $X$, associated with $(U, \varphi)$ (see [8]). It is easily seen that (4.1) represents a globally defined odd base form on $F^{*} X$; we denote this form by $\tilde{\omega}$, and call it the canonical odd $n$-form on $F^{*} X$. This form has the following properties:

(1) For each coframe field $\xi: W \rightarrow F^{*} X$, where $W$ is an open set on $X$, the pullback $\xi^{*} \tilde{\omega}$ is an odd volume form on $W$.

(2) The construction of $\tilde{\omega}$ does not depend on the orientability of the base manifold $X$. In the case of orientable and oriented manifolds $X, \tilde{\omega}$ is equivalent to an (ordinary) $n$-form on $F^{*} X$.

(3) The form $\tilde{\omega}$ is diff $X$-invariant, i.e., if $F^{*} \alpha$ denotes the canonical lift of a diffeomorphism $\alpha: W \rightarrow X$ to $F^{*} X$, then $\left(F^{*} \alpha\right)^{*} \tilde{\omega}=\tilde{\omega}$ for all $\alpha$.

It should be pointed out that odd $n$-forms $\xi^{*} \tilde{\omega}$ may be used as volume forms for integration on the base manifold $X$. In particular, these forms naturally appear as a components of Lagrangians for variational problems for coframe fields.

The canonical jet projection $\tau^{2}: J^{2} F^{*} X \rightarrow X$ is, for every $J_{x}^{2} \xi \in J^{2} F^{*} X$, defined by $\tau^{2}\left(J_{x}^{2} \xi\right)=x$. A second order Lagrangian for $F^{*} X$ is any $\tau^{2}$-horizontal $n$-form $\lambda$ defined on the second jet prolongation $J^{2} F^{*} X$ of $F^{*} X$. In the chart $\left(V^{2}, \psi^{2}\right), \psi^{2}=\left(x^{i}, z_{j}^{i}, z_{j, k}^{i}, z_{j, k l}^{i}\right)$, on $J^{2} F^{*} X$, associated with $(V, \psi)$, a Lagrangian $\lambda$ has an expression $\lambda=\mathscr{L} \cdot \tilde{\varphi} \otimes \omega_{0}$, where $\omega_{0}=d x^{1} \wedge d x^{2} \wedge \ldots \wedge d x^{n}$, $\tilde{\varphi}$ is a field of odd scalars, and $\mathscr{L}: V^{2} \rightarrow \mathbb{R}$ is the component of $\lambda$ with respect to $(V, \psi)$ (the Lagrange function associated with $(V, \psi)$ ).

We say that a second order Lagrangian $\lambda$ is natural, if for every diffeomorphism $\alpha: W \rightarrow X$, where $W$ is an open set in $X$, the canonical lift $F^{*} \alpha$ of $\alpha$ to $F^{*} X$ is an invariance transformation of $\lambda$, i.e.,

$$
\left(J^{2} F^{*} \alpha\right)^{*} \lambda=\lambda
$$


on the corresponding open set in $J^{2} F^{*} X$. An application of a general result, mentioned at the end of Section 1, to the structure considered in this paper gives us that there is a one-to-one correspondence between natural Lagrangians on $J^{2} F^{*} X$ and differential invariants $d: T_{n}^{2} L_{n}^{1} \rightarrow \widetilde{\mathbb{R}}$.

We denote by $\mathcal{A}_{\text {diff } X}^{*}$ the algebra of diff $X$-invariant functions on $J^{2} F^{*} X$. In any chart $(V, \psi), \psi=\left(x^{i}, z_{j}^{i}\right)$, on $F^{*} X$, define the functions $\mathscr{L}_{j, k}^{i}, \mathscr{L}_{j, k l}^{i}$, by

$$
\begin{aligned}
& \mathscr{L}_{j, k}^{i}=w_{j}^{s} w_{k}^{t} z_{\tilde{s}, \tilde{t}}^{i}, \\
& \mathscr{L}_{j, k l}^{i}=w_{j}^{s} w_{k}^{t} w_{l}^{u}\left(z_{\tilde{s}, \tilde{t} u}^{i}+w_{m}^{v}\left(z_{\tilde{v}, \tilde{s}}^{i} z_{\bar{t}, \bar{u}}^{m}-z_{\tilde{v}, \tilde{t}}^{i} z_{\bar{s}, \bar{u}}^{m}\right),\right.
\end{aligned}
$$

(compare with (3.1)). The functions $\mathscr{L}_{j, k}^{i}, \mathscr{L}_{j, k l}^{i}$, in coordinates expressed by (4.2), are globally well defined functions on $J^{2} F^{*} X$.

Lemma 3. (a) The functions $\mathscr{L}_{j, k}^{i}, \mathscr{L}_{j, k l}^{i}$ belong to $\mathcal{A}_{\mathrm{diff} X}^{*}$.

(b) Every function $\mathscr{L}$ from $\mathcal{A}_{\mathrm{diff} X}^{*}$ can be locally written as a differentiable function of the functions $\mathscr{L}_{j, k}^{i}, \mathscr{L}_{j, k l}^{i}$.

The following Theorem is an immediate consequence of the invariance theory.

Theorem. Every natural Lagrangian $\lambda$ on $J^{2} F^{*} X$ is of the form

$$
\lambda=\mathscr{L} \tilde{\omega},
$$

where $\mathscr{L} \in \mathcal{A}_{\mathrm{diff} X}^{*}$ and $\tilde{\omega}$ is canonical odd $n$-form of $F^{*} X$.

Remark 1. Starting with the frame action of $L_{n}^{1}$ on $L_{n}^{1}$, in the corresponding coordinates given by $\bar{p}_{j}^{i}=a_{k}^{i} p_{j}^{k}$ (compare with (1.5)), by the similar procedure it is possible to obtain differential invariants of frames. Second order differential invariants, including second order natural Lagrangians of frames, were described in [2]. Orbit reduction method gives us an alternative expression of natural Lagrangians of frames in comparison with [5].

\section{REFERENCES}

[1] N. Bourbaki, Gruppy i algebry Li, III. Gruppy Li. Moskva: Mir, 1976.

[2] J. Brajerčík, "Second order differential invariants of linear frames," Balkan J. Geom. Appl., vol. 15, no. 2, pp. 14-25, 2010.

[3] D. Q. Chao and D. Krupka, "3rd order differential invariants of coframes," Math. Slovaca, vol. 49, pp. 563-576, 1999.

[4] J. Dieudonné, Treatise on Analysis III. New York-London: Academic Press, 1972.

[5] P. L. García Peréz and J. Muñoz Masqué, "Differential invariants on the bundles of linear frames," J. Geom. Phys., vol. 7, pp. 395-418, 1990.

[6] I. Kolář, P. W. Michor, and J. Slovák, Natural Operations in Differential Geometry. Berlin: Springer-Verlag, 1993.

[7] D. Krupka, "Local invariants of a linear connection," in Diff. Geom., Budapest, 1979, Colloq. Math. Soc. J. Bolyai, vol. 31. Amsterdam: North Holland, 1982, pp. 349-369. 
[8] D. Krupka, "Natural Lagrangian structures," in Banach Center Publications, vol. 12. Warszawa: Polish Scientific Publishers, 1984, pp. 185-210.

[9] D. Krupka, "Natural variational principles," in Symmetries and Perturbation Theory, Proc. of the Internat. Conf., Otranto, Italy, 2007. World Scientific, 2008, pp. 116-123.

[10] D. Krupka and J. Janyška, Lectures on Differential Invariants. Brno: University J. E. Purkyně, 1990.

[11] U. Muench, F. Gronwald, and F. W. Hehl, "A small guide to variations in teleparallel gauge theories of gravity and the Kaniel-Itin model,” Gen. Rel. Grav., vol. 30, pp. 933-961, 1998.

\section{Authors' addresses}

\section{Ján Brajerčík}

University of Prešov, Department of Physics, Mathematics and Techniques, Ul. 17. novembra 1, 08116 Prešov, Slovakia

E-mail address: jan.brajercik@unipo.sk

\section{Milan Demko}

University of Prešov, Department of Physics, Mathematics and Techniques, Ul. 17. novembra 1, 08116 Prešov, Slovakia

E-mail address: milan.demko@unipo.sk 